Itinéraires Itinéraires

Littérature, textes, cultures

2018-1| 2018

Le manifeste à travers les arts : devenirs d'un genre indiscipliné

\title{
Du mélos en manifeste
}

Melodicon Manifesto

\section{David Christoffel}

\section{OpenEdition}

Journals

Édition électronique

URL : http://journals.openedition.org/itineraires/4421

DOI : 10.4000/itineraires.4421

ISSN : 2427-920X

Éditeur

Pléiade

Référence électronique

David Christoffel, «Du mélos en manifeste », Itinéraires [En ligne], 2018-1 | 2018, mis en ligne le 15 septembre 2018, consulté le 16 mai 2020. URL : http://journals.openedition.org/itineraires/4421 ; DOI : https://doi.org/10.4000/itineraires.4421

Ce document a été généré automatiquement le 16 mai 2020

\section{(c) () $९$}

Itinéraires est mis à disposition selon les termes de la licence Creative Commons Attribution - Pas d'Utilisation Commerciale - Pas de Modification 4.0 International. 


\title{
Du mélos en manifeste
}

\author{
Melodicon Manifesto
}

David Christoffel

1 Dans Manifesto (2015), Julian Rosefeldt part de différents manifestes (futuriste, dadaïste, situationniste, etc.) pour procéder à autant de déplacements énonciatifs que de personnages qu'il fait interpréter par Cate Blanchett: une mère de famille, une riche dame qui jette quelques généralités sur l'art aux invités d'un cocktail dans sa très belle maison, une enseignante d'école primaire qui, au lieu de bonnes vieilles leçons de chose, enseigne à ses élèves les préceptes de Dogma. Le ton péremptoire ou solennel des manifestes collectionnés est alors ressaisi en autant de situations quotidiennes où la parole est appelée à généraliser : divagations en pleine soirée décadente, oraison funèbre, prière familiale, dialogue entre journaliste de plateau et journaliste de terrain, etc. Comme s'il fallait collectionner ces moments de rituels discursifs pour pouvoir projeter dans les prises de parole quotidiennes, quelques considérations plus fondamentales. En quelque sorte, Julian Rosefeldt répète une certitude benjaminienne : le journalisme généralisé place la civilisation dans une crise du récit qui distribue une sorte d'hypertrophie de toute prise de parole. Nous verrons que cette manière de rejouer l'affirmation manifestaire revient à produire une "performance de la revendication esthétique ", tout en problématisant le rapport entre l'affirmation de nouvelles valeurs esthétiques (ou méta-esthétiques ou post-esthétiques) et la question de la valeur de l'art.

2 À l'époque où les histoires entre Anciens et Modernes étaient traitées dialectiquement, on s'entendait sur le fait d'une différence de nature entre jugement déterminant et jugement réfléchissant (cf. Kant [1790] 1995 : §58, 308). L'œuvre d'art pouvait s'en prévaloir pour justifier d'un statut privilégié dans l'intimité spécifique qu'elle tenait entre son fond et sa forme. Tandis qu'un idéal révolutionnaire ne saurait arraisonner une production artistique sans universaliser suffisamment ses motifs pour que sa fin arrive à justifier des œuvres d'art plus décisives que de simples moyens ${ }^{1}$. Autrement dit, il y aurait moins de chances de renverser l'ordre établi en commençant par canaliser les espoirs de renversement sur le fond un peu plus que dans la forme. Si une œuvre doit être précédée d'un manifeste pour attester la dimension révolutionnaire de 
son contenu, s'il lui faut se montrer révolutionnaire d'une deuxième façon par sa forme, alors nous ne parlons plus vraiment d'une œuvre d'art, mais d'un discours apologétique (Adorno [1970] 1974 : 321).

3 Sans avoir à aller jusque-là, nous comprenons déjà que la liaison entre manifeste et musique est très dépendante du cadre esthétique dans lequel nous envisageons de la penser. Notre intérêt à historiciser le traitement dialectique de la question est donc celui-ci : c'est au moment où la musique viendrait à se définir au plus intrinsèquement comme un art capable de renouvellements délibérés, consignés en manifestes, que le concept de révolution se trouverait lui-même bouleversé. En ce sens, nous devrons au plus vite baliser en quoi la musique réagit si spécifiquement à la notion de manifeste, pour ne pas nous trouver confrontés à l'inversion de la question - même s'il serait plus improbable de chercher en quoi la révolution pourrait être une activité intrinsèquement musicale... Sauf à considérer que tout commence mieux par là.

\section{L'idéalisation poétique du révolutionnaire musical}

Quand la philosophie des Lumières est réputée cause finale de la Révolution française, il est supposé une analogie entre le criticisme kantien (comme sécularisation de l'entendement corollaire d'une "absolutisation" de l'esthétique) et le surgissement d'un égalitarisme républicain à la place d'un ordre "géocentré » de droit divin. La même analogie peut fonctionner entre l'esprit révolutionnaire de 1789 et le romantisme allemand. L'analogie résonne à deux niveaux au moins dans la philosophie hégélienne. D'une part, la critique de la belle âme, des efforts individuels comme obstruction par rapport à l'idéal (Hegel 1979: 210-211) ${ }^{2}$. D'autre part, l'appel au dépassement de la conception kantienne qui, pour Hegel, est une sorte de fin de nonrecevoir d'un Beau juché jusqu'au-delà de la raison pratique. Même, le Beau romantique est justement révolutionnaire parce qu'il ne se laisse pas traduire en concept. Nous pourrions ainsi argumenter que la promotion de la "musique absolue » par le romantisme est une affirmation révolutionnaire, mais aussi une essentialisation : il s'agit d'affirmer la musique comme un art révolutionnaire, intrinsèquement révolutionnaire (pour autant que l'expression «intrinsèquement révolutionnaire " bute sur un essentialisme hors sujet) ; au point que la défiance à l'instrumentalisation politique de l'art n'a peut-être jamais été aussi nette.

D'où l'idée de tenir pour significatif qu'en amont de ce moment précis de l'histoire, la production de musique instrumentale prenne le pas sur la musique vocale. En relevant que Mozart a renversé la proportion traditionnelle entre le répertoire chanté et la musique instrumentale, Marc Vignal ${ }^{3}$ laisse entendre que le compositeur emblématique du classicisme musical est en fait un préromantique. Il en ressort des ambiguïtés constitutives : à l'époque où la musique affirme activement son autonomie à l'égard des autres arts $^{4}$, elle cherche à nouer avec la poésie une égalité de hauteur ontologique ${ }^{5}$. Nous verrons comment Nietzsche réinvestit les charges révolutionnaires de la musique dans ses écarts avec la poésie. Plus la musique la porte en propre, plus la notion de révolution s'en trouve certes rehaussée, mais d'abord transformée. Au-delà des rapports entre la musique romantique, les autres arts et la remise en question des formes et genres classiques (démultiplication du nombre des mouvements des symphonies par Beethoven, émancipations hétéronomes des miniatures par Schumann, etc.), ce sont aussi les liens de la musique avec les enjeux socio-politiques qui 
deviennent équivoques à l'âge romantique. L'importance que le romantisme donne à la musique dite « absolue » tient au privilège qu'il entend donner à sa force poétique : « Ce que Tieck appelle "poétique" se nomme "romantique" chez Hoffmann, et ce qui est "purement romantique" se manifeste dans ce qui est "véritablement musical" " (Dahlhaus 1997 : 64).

6 Dans son essai dédié à $L a$ Musique romantique, Alfred Einstein renvoie aux notions d'universalisme et de nationalisme. Si elles sont rationnellement incompatibles, leur incompatibilité est d'autant plus criante quand il s'agit d'en débattre politiquement, tandis qu'elle semble le support d'une contradiction esthétique fructueuse. Dans une chronique de Heine, écrite à Paris le 4 février 1838 pour la Gazette d'Augsbourg, nous lisons :

Chopin est né en Pologne de parents français et a reçu une partie de son éducation en Allemagne. Les influences de ces trois nationalités donnent à sa personne quelque chose de singulièrement remarquable ; il s'est approprié, en effet, tout ce qui distingue en bien ces trois peuples: la Pologne lui a donné son sens chevaleresque et sa douleur historique, la France sa grâce légère et son charme, l'Allemagne sa profondeur romantique... (Heine [1838] 1997 : 38 et suiv.)

7 Ce qui voudrait dire que l'âme est un lieu de poésie de la même façon qu'un pays est une valeur sinon morale du moins esthétique, qu'une langue est une sorte d'auberge et, par suite, qu'un compositeur est comme un porte-drapeau et sa musique comparable à une « veste " pour collectionner les blasons. Heine ne tient pas vraiment le polonais, le français et l'allemand pour des pôles linguistiques. Il s'y réfère comme à des traits de caractère, des valeurs spécifiques. Or, au lieu d'interpréter les différences culturelles entre les peuples en termes trop séparés (et tout de même patriotiques), il envisage un lieu de l'exil, indépendant de ses repères géographiques, se réclamant de la poésie pour qualifier ce qu'il peut y avoir d'universalité dans la musique : «[...] quand il s'assied à son piano et qu'il improvise. Il n'est alors ni Polonais, ni Français, ni Allemand; il trahit une origine bien plus haute, il descend du pays de Mozart, de Raphaël, de Goethe : sa vraie patrie est le royaume enchanté de la poésie» (Heine [1838] 1997: 38). Si la musique est constituée comme une source de poésie, c'est qu'il faut s'engager, avec Heine, dans une vision universalisée de la poésie. C'est à ce titre que la musique peut subsumer les patriotismes, en se qualifiant de poétique. C'est par la force de ce nouvel ordre d'absolu qu'elle entend se vouloir révolutionnaire et se traduire comme manifeste d'une nouvelle importance de l'engagement par la musique.

Quand, en 1894, Saint-Georges de Bouhélier publie dans la revue L'Académie française, "Thème à variations - Notes sur un art futur» (que Michel Decaudin qualifie de manifeste dans La Crise des valeurs symbolistes), on comprend que la métaphore musicale de "Thème et variations » appliquée au domaine des idées esthétiques est significative. Si ces notes se signalent comme autant de "variations " sur le modèle musical, c'est que la forme éparse de la réflexion doit valoir pour revendication prospective attachée à cet éclatement. Il faut entendre par là que le caractère discontinu ou/et fantaisiste de ces variations est susceptible de faire varier le statut de l'art lui-même et, partant, son devenir. Ce qui pourrait impliquer que toutes les écritures musicales discontinues ou/ et fantaisistes doivent s'entendre comme des écritures manifestaires, en particulier du point de vue des ruptures de responsabilité que suppose l'abandon de la continuité. Or, c'est exactement ce que semblent revendiquer les manifestes bruitistes qui, annonçant que ces esthétiques sonores ne peuvent en aller sans revendication manifestaire préalable, obligent à une forme de jusqu'au-boutisme : aucune dimension de leur art ne 
pourra échapper à leur revendication, le moindre bruit devra être reconnu dans sa dignité musicale. C'est pourquoi les musiques qui supposent un préalable manifestaire, supposent aussi une redistribution du rapport entre la pratique et la théorie. La continuité est alors frappante de Luigi Russolo à Pierre Schaeffer: si tous les sons méritent attention, c'est que l'effort théorique pour élargir le champ des objets musicaux est indispensable et très attaché à la pratique de la musique bruitiste, puis concrète.

\section{Révolutionnaire et musical à la fois ?}

9 À considérer qu'il y ait des musiques plus ou moins révolutionnaires, la révolution deviendrait une valeur musicale. Est-ce à dire qu'une révolution musicale doit se distinguer des autres manières de faire révolution? On ne peut aller jusqu'à valider l'idée qu'il y a autant de types de révolution que de domaines d'activité et considérer qu'on peut faire la révolution en musique comme on peut la faire en médecine, en athlétisme, en broderie, etc. Un peu comme dans l'Hippias mineur ${ }^{6}$, on entend bien le piège des définitions privatives de la révolution : est-on conservateur quand on n'est pas ému par la Carmagnole? L'auteur de la Carmagnole était-il nécessairement un bon révolutionnaire? La confection de bonnets phrygiens n'est-elle pas une activité plus utile à la révolution que l'écriture de chansons? Si l'attribut révolutionnaire n'a pas la même vocation selon qu'il est employé au sujet d'une personne ou d'une forme artistique, il faut dissoudre le paradoxe selon lequel un compositeur révolutionnaire pourrait faire de la musique réactionnaire (comme il est encore courant de voir des individus socialement libertaires produire des textes poétiquement conservateurs). Dans Musique et société du second empire aux années vingt, Michel Faure (1985) intitule un chapitre « Esthétique et idéologies " pour montrer qu'il y a presque autant de manières d'être révolutionnaire qu'il y a de compositeurs et d'autant plus que les ambiguïtés sont assez nombreuses pour chacun. Les titres des quatre parties du livre sont sur ce point exemplaires :

« A. Saint-Saëns : républicanisme de combat et éclectisme parnassien »,

«B. Fauré : opportunisme républicain et esthétique de la distanciation »,

«C. Debussy : nationalisme monarchique et sacralisation de l'art ",

«D. Ravel : anarcho-bolchevisme et esthétique du pastiche ».

Sous cet angle sociologique, la question pourrait être d'identifier à quelle époque, dans quel contexte, par quelle classe sociale a pu prendre consistance et autonomie l'idée même qu'une forme, indépendamment des positionnements sociaux de son auteur, puisse être révolutionnaire. Cela revient à considérer telle œuvre musicale du point de vue d'une revendication esthétique, à la tenir pour une sorte de manifeste affirmant, en plus de sa forme, la prescription de celle-ci comme nouvelle manière, même si la révolution comme modèle de dynamique compositionnelle peut s'entendre elle-même de façons très variées. Révolutions musicales, l'ouvrage de Dominique et Jean-Yves Bosseur, montre ainsi que la musique peut "se révolutionner" par chacune de ses composantes. De ce point de vue, on peut considérer que Schönberg agit en révolutionnaire sur le paramètre harmonique, tandis que Nancarrow dépasse les frontières de l'humainement exécutable, autant de ruptures de valeur esthétique qui suffisent en général à les tenir pour des révolutionnaires du langage musical. Il y a, dès lors, toujours quelque chose de réducteur à mettre toutes ces ruptures en équivalence au nom d'une dynamique révolutionnaire virtuellement comparable. Dominique et 
Jean-Yves Bosseur tentent de dissiper la réduction par un ordonnancement quasiment hiérarchique, présentant le principe cagien d'indétermination comme une résolution, presque un dépassement dialectique (dynamiquement finaliste, finaliste quand même $\mathrm{P}^{7}$ ) des révolutions auxquelles il donne suite. Or ce qui nous empêche de mettre toutes les révolutions musicales au même niveau, de mettre sur un pied d'égalité de valeur par exemple la «dissolution de la tonalité » par Schönberg et l'éclatement de la notion d'œuvre par Cage (le manifeste Bang Fist devant être tenu pour une œuvre du compositeur), c'est justement qu'ils reconfigurent respectivement la notion de révolution en musique et, par extension, la révolution en soi.

11 D'une part, Dominique et Jean-Yves Bosseur montrent comment Cage révolutionne, par exemple, le pouvoir de l'interprète sur la forme :

Le fait qu'il n'y ait pas, dans sa musique, de cohérence logique réglant les rapports entre la structure compositionnelle et la forme résultante favorise l'éclatement de l'œuvre ; celle-ci n'est plus un objet clos mais un organisme dynamique qu'il revient à l'interprète de faire vivre, et pas seulement d'achever. (Bosseur 1999 : 57)

D'autre part, Esteban Buch ouvre son "histoire de la grandeur de Schönberg» en montrant comment son objet induit une refonte des habitudes musicologiques :

La valeur d'une œuvre d'art n'est jamais acquise ; seule son importance historique peut l'être. Le cas Schönberg est un exemple frappant de la dynamique sociale du jugement esthétique, qui fait des œuvres artistiques ou littéraires l'enjeu d'interprétations sans cesse renouvelées ou bien de configurations idéologiques évolutives et diversement conflictuelles. (Buch 2006 : 11)

Nous en arrivons au paradoxe: pour être un révolutionnaire, il faut être un grand compositeur et avoir conquis cette grandeur sur des critères non nécessairement révolutionnaires (puisqu'il s'agit d'une grandeur socialement admise, nous supposons qu'elle repose justement sur des critères préalables à la rupture supposée). Autrement dit, nous restons dans un paradigme romantique où il n'y a pas révolution sans héroïsme. L'art ne fait pas exception au préalable de la grandeur. Et c'est encore à ce prix que l'art peut être conjoint à un mouvement révolutionnaire. Si bien que les ruptures sont devenues les discriminants de l'évolution de l'histoire de la musique parce qu'elles sont, d'abord, des critères d'interprétation de l'histoire générale. Nous pourrions même avancer que la musique au $\mathrm{xx}^{\mathrm{e}}$ siècle évolue par des révolutions d'un autre ordre que celui des ruptures $d u \mathrm{XIII}^{\mathrm{e}}$ ou XVIII ${ }^{\mathrm{e}}$ siècle, en vertu du fait que l'Idée de révolution a elle-même évolué $\mathrm{du} \mathrm{XIII}^{\mathrm{e}}$ au $\mathrm{xx}^{\mathrm{e}}$ siècle. Nous pourrions aussi en conclure que la musique fait ses révolutions suivant des schémas qui, pour être reconnus révolutionnaires, ne sont pas strictement musicaux. Au même titre, nous pouvons aussi soutenir que la musique peut modifier l'Idée de révolution de manière spécifique. Dès lors, à titre post-dialectique, le $\mathrm{xx}^{\mathrm{e}}$ siècle permet, par l'écriture de manifestes, de thématiser une esthétique de la revendication (Boulez) et par l'élargissement du domaine de la composition à des formalisations manifestaires créatives (Nam June Paik), de concevoir une performance de la revendication esthétique (George Brecht).

\section{Si la musique révolutionne la notion de révolution}

L'idée de révolution n'ayant ni la même valeur ni la même dynamique selon qu'elle est envisagée dans le champ politique ou dans le domaine esthétique, il nous reste à considérer comment ces différents champs se trouvent réarticulés par une acception 
plus générale (cosmologique ?) de la révolution. Wagner ouvre L'Art et la Révolution ([1849] 2001) en posant la différenciation des arts (danse, musique, poésie, arts plastiques) comme concomitante de la désagrégation de l'œuvre d'art universelle dont la tragédie grecque est le modèle. Alors que nous avons laissé entendre que la Révolution française pouvait trouver quelques aspects en commun avec le criticisme kantien et, par un même type d'analogie, avec le romantisme musical (avec l'ambiguïté entre la prépondérance de la musique instrumentale - de Beethoven à Mozart, si l'on peut dire - et l'éclatement relatif mais significatif de la forme - Schubert et Schumann), nous devons reconnaître que la dimension révolutionnaire est modifiée par l'élan de réunification des arts et comme relancée par la préconisation wagnérienne d'entendre la musique servir le drame (au point de composer des opéras intégralement chantés, sans récitatif). Le paradoxe voudrait ici que la musique soit d'autant plus révolutionnaire qu'elle serait dramatique. Et d'autant mieux dramatique qu'elle serait autonome. Cette liaison de la musique à sa puissance dramatique intrinsèque peut rappeler la tragédie grecque et la thèse de Nietzsche pour qui le dualisme apollinien / dionysiaque ne constitue pas un clivage politique (il ne s'agit pas de dire que Dionysos est le seul révolutionnaire des deux), notamment parce qu'il est diachronique. Dans $L a$ Naissance de la tragédie, Nietzsche distingue différents âges dans l'Antiquité grecque, autant de niveaux dans la "dédionysation ${ }^{8}$ " de la poésie: "[...] nous pouvons distinguer deux courants principaux dans l'histoire de la langue grecque, selon que le langage imite le monde des phénomènes et des images ou bien le monde de la musique » (Nietzsche [1872] $1977:$ 49). Et c'est bien dans cette tendance à une sorte de stricto-dionysisme que Nietzsche semble redouter la part de musique absolue :

Une symphonie de Beethoven, on en fait l'expérience tous les jours, contraint chaque auditeur à un discours imagé, même si le rapprochement des différents mondes d'images suscités par un morceau de musique donne un résultat parfaitement bariolé et fantastique, voire contradictoire. (Nietzsche [1872] 1977 : 49)

Nous pourrions en déduire que la musique est révolutionnaire et contient une force manifestaire partout où elle est absolue, dionysiaque et excessivement détachée de la poésie. Entendu dans tous ces termes, nous pouvons supposer qu'elle est relativement rare. Nous pourrions même poser que la musique majoritaire est au contraire conventionnelle et très attachée à la poésie. Plus tard, Nietzsche envisage même que la musique instrumentale puisse être assez couramment poétique :

La «musique absolue» est ou bien une forme en soi, au stade rudimentaire de la musique où le plaisir naît tout bonnement de sons produits en mesure et d'intensités diverses, ou bien le symbolisme des formes dont la langue est comprise même sans poésie, après une évolution dans laquelle les deux arts furent unis jusqu'à ce qu'enfin la forme musicale fût entièrement entretissée de fils d'idées et de sentiments. (Nietzsche [1878] $1968: 164-165)$

C'est bien à partir de là que la perspective d'un langage musical révolutionnaire prend une nouvelle tournure. Il ne s'agit plus de chercher à quelle révolution la musique peut bien être associée, mais en quoi la musique est en elle-même une activité en prise avec le temps historique et le contexte qui la relie à telle ou telle révolution. Adorno revient sur ce fragment de Nietzsche et souligne le fait que la musique ne se laisse pas décomposer en illusion et réalité. Pour Adorno ([1948] 1962 : 148), Nietzsche pense de façon trop « mécanique » la séparation entre le son et ce qui y est « introduit ». Et alors qu'il accuse Nietzsche de concevoir «de façon trop linéaire le progrès musical en l'identifiant à une psychologisation croissante " (Ibid.), Adorno tire davantage de la 
dialectique en considérant que " le matériau par lui-même est déjà esprit » (et, partant, toute œuvre pourrait valoir pour manifeste de sa manière). En évoquant la psychologie musicale d'Ernst Kurth, Adorno l'accuse de nier « l'élément hétérogène et matériel du son ». Adorno fait cette mise au point philosophique à l'occasion d'une argumentation sur le refus d'authenticité par Stravinsky. Nous n'entendons pas ici répondre du dédoublement de la révolution en musique (la révolution du langage musical à la place de la révolution sociale avec relais musicaux). Pour justifier que la question soit dialectisée, nous constatons que son dédoublement nécessitait d'être formalisé. Mais surtout, une fois passées les vapeurs idéologiques des révolutions musicales diffusées dans l'ordre politique, nous pouvons voir des courbures hétérogènes et le relatif absolutisme romantique se traduire de façon beaucoup plus réactionnaire que révolutionnaire :

Les «Lumières » ont légué au romantisme plébéien l'idée de la continuité de la Création, du minéral à l'humain en passant par le végétal et par l'animal. Et ce romantisme humanitaire a versé dans la métempsycose pour rétablir la circulation sur toute l'étendue de l'échelle sociale. (Faure 1985 : 203)

17 Adorno reprend à Cocteau l'idée que Le Sacre du printemps est une révolution de type fauviste (Cocteau 1918 : 64, cité par Adorno [1948] 1962: 147), avec ce que cela suppose de générationnel, c'est-à-dire de généalogique, de continuiste : «L'élève de ce RimskyKorsakoff qui corrigeait l'harmonie de Moussorgsky selon des règles de conservatoire, s'est insurgé contre les ateliers, comme seulement un fauve dans la peinture » (Adorno [1948] 1962 : 147). Mais au-delà d'une rupture de style, Stravinsky marque une rupture avec la question du style. Ce que nous présentions comme une différence d'acceptions entre révolution formelle et révolution sociale se trouve alors réinvestie, à considérer que le langage musical incorpore son époque (comme Nietzsche disait que telle tonalité ou telle tournure a pu devenir le signifiant de telle ou telle idée). Adorno reprend l'argument de Nietzsche (l'arbitraire du contenu - par exemple révolutionnaire - du signe, qui plus est quand il est musical), en même temps qu'il tente de le redéployer d'une manière quasi méta-historique :

Stravinsky a dédaigné le chemin facile vers l'authenticité ; cela eût été le chemin de l'académisme, à savoir la limitation au répertoire approuvé de l'idiome musical élaboré durant le XVIII ${ }^{\mathrm{e}}$ et le $\mathrm{XIX}^{\mathrm{e}}$ siècle et qui a acquis le cachet du «naturel » et de ce qui va de soi, pour la conscience bourgeoise à laquelle elle appartient. (Adorno [1948] 1962 : 145)

18 Dès lors, le rejet de la mélodie continue wagnérienne, la superposition de registres musicaux hétérogènes (enfantins, populaires, tribaux, etc.), le recours à des instrumentations " pluriculturels » peuvent être interprétés comme autant de marques formelles d'une "esthétique du discontinu». Dans Adorno et la nouvelle musique, Raymond Court montre qu'en relevant les spécificités stylistiques de l'anti-stylisme de Stravinsky, Adorno semble faire des motivations anti-psychologiques, nihilistes, infantilistes, du compositeur les facteurs d'un «style du brisé », mais d'un style quand même. Or l'étiquette (synthétique et ramassée comme une étiquette) de « esthétique de la discontinuité » pourrait donner l'impression que la discontinuité peut devenir en elle-même un critère esthétique, ou même une valeur plus ou moins révolutionnaire. Nous avons déjà eu l'occasion de traiter de la discontinuité en musique (Christoffel 2007), et avons distingué la discontinuité comme catégorie (formelle, théorique) de la discontinuité comme critère (distinctif, axiologique). Or, qu'une musique soit révolutionnaire par son contenu ou son usage (comme peut l'être la Carmagnole), ou par 
sa facture ou son inscription historique (Schönberg, Stravinsky), sa valeur révolutionnaire demeure extrinsèque. Tout en sortant des clôtures d'une axiologie de l'avant-garde, Martin Kaltenecker suggère que les révolutions musicales - peut-être plus que les révolutions sociales -, sont des marqueurs historiques. Le musicologue reprend la distinction de Paul Veyne entre l'histoire pure et l'histoire axiologique (1978: 94-99) qui met l'emphase sur l'importance historique des œuvres plutôt que sur les œuvres elles-mêmes (en tant que forme), au point de projeter l'art dans une idée de progrès. D'où le fétichisme tout spécial dont les manifestes peuvent faire l'objet. Car soutenir qu'une œuvre est révolutionnaire, c'est en même temps l'envisager dans un horizon qui la dépasse et valoriser qu'elle s'en soit arrachée. Et il y a maintenant quelque chose de "vingtiémiste » (de séculaire plus que de sécularisant) à ce que les œuvres procèdent par rupture avec un goût dominant. Martin Kaltenecker préfère la catégorie de l'interruption pour corréler cette rupture à un ton particulier et au fait que les œuvres «s'interrogent elles-mêmes, nous communiquant la conscience de leur propre précarité, d'un doute » (Kaltenecker 2000). Mais c'est aussi la limite de ce que nous pouvons reconnaître de révolutionnaire dans des œuvres musicales, tant que nous les considérons dans l'en-soi de leur forme.

\section{Potentialités révolutionnaires de la technologisation de la musique}

19 Si nous relevons par exemple que les écrits de Walter Benjamin sur la photographie s'inscrivent à l'époque d'une crise de la perception, nous pouvons aussi nous rappeler que la pratique radiophonique du philosophe entend apporter une réponse à la crise de la narration (Baudouin 2009). Avant qu'Adorno n'écrive De l'utilisation musicale de la radio en 1938-1939, Rudolf Arnheim a publié Radio en 1936 où la question est soulevée de musiques plus ou moins "radiogéniques». Que la radio puisse formater la production musicale, exiger des compositeurs qu'ils intègrent à la facture de leurs œuvres les usages les plus appropriés pour que leurs œuvres soient les plus radiogéniques, c'est édifier l'antagonisme entre diffusion (popularité) et liberté d'expression. Dans l'Allemagne des années 1920, quand Arnheim réfléchit à l'impact de la radio sur l'expression, les usages propagandistes de la radio sont aussi les moins adaptés au médium, en ce qu'ils utilisent le son de façon mimétique et naturaliste, pour reprendre les catégories isolées par Martin Kaltenecker (2005: 25). Seulement, à la différence du cinéma et de la photographie, " la radio ne se définit pas a priori comme un art » (Baudoin 2009:58) pour autant qu'elle pose, comme tous les nouveaux médias qui lui sont contemporains, la question de la reproductibilité technique. La musique trouve dans sa manifestation radiophonique un nouveau régime de diffusion, peut alors s'interroger sur son statut social et redéfinir les modalités d'interpellation en son pouvoir à l'égard de ses auditeurs.

Aussi, en considérant Stravinsky du point de vue de la «crise sans précédent des langages musicaux» (Court [1981] 2007: 66), il faut saisir comment les révolutions musicales apparaissent au sein de révolutions plus larges (que strictement sociales). Il est donc maintenant question de pointer quelle place occupe la musique dans une crise du signe et, qui plus est, dans les perspectives révolutionnaires auxquelles elle appelle. «Le signe signifie, alors que la forme se signifie » (ce qui laisse entendre que tout est manifeste!). En repartant de cette formule de Focillon (Ibid.: 71), Raymond Court 
raccroche la musique au statut tellement particulier qu'elle occupe dans l'accès de Jakobson aux affaires poétiques: «[...] une analyse comme celle de Jakobson nous permet de saisir comment le linguistique se convertit au poétique au moment précis où le procès métaphorique passe au premier plan en réinvestissant le procès métonymique lui-même " (Ibid. : 73). C'est en saisissant la forme constituée par l'événement de son exécution et en cherchant à échapper à quelque fonctionnalisation que la musique parvient à renouveler son débat formel et à se saisir comme objet d'un monde médiatisé, virtualisé. C'est ainsi que la typologie proposée par Costin Miereanu dans Fuite et conquête du champ musical, entre les musiques minimales, les musiques indéterminées, les musiques répétitives, les musiques transitives et le Poly-art, donne aux transgressions de forme une valeur de requalification du statut de l'objet musical.

Des multimédia, le Poly-art gardera le désir de mettre en présence divers domaines de la recherche artistique dans une relation de complémentarité, afin de configurer un espace-son-spectacle syncrétique situé aux confins de plusieurs disciplines artistiques dépendantes du temps, de l'espace, ou des deux réunis. (Miereanu 1995 :

68)

21 L'appartenance à telle ou telle catégorie peut alors toucher à la manière dont une œuvre peut acquérir une charge manifestaire, sans l'y conditionner nécessairement. En bref, ce n'est pas parce qu'elle est musicale qu'une œuvre est révolutionnaire. C'est plutôt pour l'être mieux qu'elle peut se charger d'une dimension de manifestaire. Le passage de la musique d'œuvre d'art à "contenu » médiatique peut se lire de deux façons au moins. D'abord, elle semble ouvrir le débat d'une manière toute cinglante, la musique d'ameublement de Satie a effectivement été d'avant-garde pour ce qu'elle anticipait, en l'outrant, le devenir-industriel de la musique. Mais ensuite, l'ambivalence peut aussi jouer dans l'autre sens : si la musique se saisit comme objet médiatique, sa capacité à signifier doit se ressaisir avec des outils analytiques spécialement adaptés à son nouvel âge technologique. Quand les chercheurs du MIM9 ont posé les Unités Sémiotiques Temporelles comme "éléments nouveaux d'analyse musicale", ils ont donné à la musique une puissance de signifiance applicable à tout régime de signe. Pour Xavier Hautbois (2004), la sémiotique temporelle est universelle et la psychologie cognitive est en train de confirmer ${ }^{10}$ que les UST correspondent aux représentations mentales des enfants très jeunes à partir de l'expérience corporelle du mouvement (Zénouda 2008 : 289). Réparties en six catégories (trois variants, trois invariants), les UST couvrent le champ perceptif du mouvement et, en quelque sorte, peuvent permettre de catégoriser toutes les tendances politiques. Les trois «invariants par stagnation" (En suspension, Stationnaire, En flottement) peuvent faire penser aux forces conservatrices. Les trois "variants à évolution uniforme" (Qui avance, Trajectoire inexorable, Lourdeur) font penser aux réformismes. Il est alors intéressant de noter que nous ne saurions trop quelle couleur politique donner aux « invariants par répétition» (Par vague, Qui tourne, Obsessionnel) et aux "variants à évolution contrariée » (Sur l'erre, Freinage, Étirement, Qui veut démarrer). Mais surtout, il resterait à définir si les révolutionnaires sont plutôt les mouvements "variants à équilibre rompu» (Chute, Élan, Contracté-étendu, Suspension-interrogation) ou les «invariants par effet chaotique » (Sans direction par divergence d'information, Sans direction par excès d'information), et pourquoi la charge qui fait d'une œuvre un manifeste ne saurait ainsi être rapportée à nouveau à des critères aussi formels. 


\section{BIBLIOGRAPHIE}

Adorno, Theodor W., [1948] 1962, Philosophie de la nouvelle musique, Paris, Gallimard, coll. « Tel ».

Adorno, Theodor W., [1970] 1974, Théorie esthétique, traduction Jimenez, Paris, Klincksieck.

Baudouin, Philippe, 2009, Au microphone: Dr Walter Benjamin, Paris, Éditions de la Maison des Sciences de l'Homme, coll. «Philia».

Bosseur, Dominique et Jean-Yves, 1999, Révolutions musicales, Paris, Minerve.

Buch, Esteban, 2006, Le cas Schönberg, Paris, Gallimard.

Christoffel, David et Guibert, Daniel, 2009, Dialogique pour L'origine des individus, Paris, Éditions Criticalsecret.

Christoffel, David, 2007, « Le grain n'est pas le sujet », Discontinuité, Variations, nº 15, p. 117-132.

Court, Raymond, [1981] 2007, Adorno et la nouvelle musique, Paris, Klincksieck.

Dahlhaus, Carl, 1997, L'idée de la musique absolue, Genève, Contrechamps.

Einstein, Alfred, [1959] 1989, La Musique romantique, Paris, Gallimard, coll. « Tel ».

Faure, Michel, 1985, Musique et société du second Empire aux années vingt, Paris, Flammarion, coll. « Harmoniques ».

Hautbois, Xavier, 2004, « Les Unités Sémiotiques Temporelles : de la sémiotique musicale vers une sémiotique générale du temps dans les arts ", Gestes, formes et processus signifiants en musique et sémiotique interarts, ICMS 8, Paris.

Hegel, Georg Wilhelm Friedrich, 1979, L'Idée du Beau, Paris, Flammarion, coll. « Champs ».

Heine, Heinrich, [1838] 1997, Mais qu'est-ce que la musique?, Arles, Actes Sud.

Kaltenecker, Martin, 2000, « “Intrigue” et “Interruption”. Programme pour une histoire de la musique contemporaine », Critique, $\mathrm{n}^{\circ}$ 639-640, « Musique(s) Pour une généalogie du contemporain ", p. 777-789.

Kaltenecker, Martin, 2005, «La retouche du réel - l'esthétique radiophonique de Rudolf Arnheim », dans Rudolf Arnheim, Radio, Paris, Van Dieren.

Kant, Emmanuel, [1790] 1995, Critique de la faculté de juger, Paris, Gallimard, coll. « Folio essais ».

Miereanu, Costin, 1995, Fuite et conquête du champ musical, Paris, Klincksieck.

Nietzsche, Friedrich, [1878] 1968, Humain, trop humain. Unlivre pour les esprits libres I, § 215, Paris, Gallimard, coll. « Folio essais ».

Nietzsche, Friedrich, [1872] 1977, La Naissance de la tragédie, Paris, Gallimard, coll. « Folio essais ». Socrate à Hippias, Hippias mineur, X, traduction Émile Chambry, [En ligne], https:// fr.wikisource.org/wiki/Hippias_mineur_(trad._Chambry).

Veyne, Paul, 1978, Comment on écrit l'histoire, Paris, Seuil, coll. « Points ».

Vignal, Marc, 2001, Haydn et Mozart, Paris, Fayard.

Wagner, Richard, [1849] 2001, L'art et la révolution, Mons, Sils Maria, coll. « Les immanents ». 
Zénouda, Hervé, 2008, Les images et les sons dans les hypermédias artistiques contemporains, Paris, L'Harmattan, coll. « Recherche d'universaux et langages a-média ».

\section{NOTES}

1. «L'objectivité extérieure, pour autant qu'elle est la réalité de l'idéal, doit se dépouiller de sa rudesse et renoncer à son indépendance objective pour se révéler identique à ce dont elle est la manifestation extérieure » (Hegel 1979 : 322).

2. III, A, 1 «La belle individualité » (qui rend tellement paradoxale l'incrimination de la figure échevelée du romantisme, comme pour d'autant mieux en révéler la futilité).

3. «Les œuvres principales écrites à Salzbourg entre les deux derniers séjours en Italie furent toutefois huit symphonies [...]. Six ont quatre mouvements, seules celles en ut majeur $n^{\circ} 16 \mathrm{KV}$ 128 et en sol majeur $n^{\circ} 17 \mathrm{KV} 129$ (mai 1772) se limitent à trois. Mozart voulut-il par cette production abondante impressionner son nouveau patron Colloredo? Sûrement pas. C'était plutôt de sa part une provocation, car à Salzbourg, la principale obligation d'un compositeur de cour était de composer non des symphonies, mais des musiques d'église » (Vignal 2001 : 116).

4. «[...] si le Romantisme considère la musique comme le centre, le germe et l'origine première de tous les arts, il voit aussi dans la musique purement instrumentale le centre de la musique tout entière, et ce justement en raison de sa prétendue imprécision et de la diversité d'interprétation dont elle est, selon eux, susceptible » (Einstein [1959] 1989 : 32).

5. « [...] le Romantisme a été le premier à réaliser l'idéal du lied, c'est-à-dire la parfaite unité du texte et de la musique, l'équilibre absolu du chant et de l'accompagnement » (Einstein [1959] $1989: 89)$.

6. «Mais voici ce que je veux dire : considère les arts que tu possèdes - et ils sont en nombre suffisant - et les arts des autres, et dis-moi si, d'après ce que nous avons admis d'un commun accord, tu en vois un seul où l'homme véridique se distingue du menteur et où ils ne soient pas le même homme » (Socrate à Hippias, Hippias mineur, X, traduction Émile Chambry, Wikisource).

7. Sur la dialectique comme finalisme dynamique, cf. Christoffel et Guibert (2009).

8. C'est-à-dire la rationalisation et la dramatisation, pour reprendre l'argumentation de Nietzsche dans La Philosophie à l'époque tragique des Grecs.

9. Laboratoire Musique et informatique de Marseille (http://labo-mim.org/).

10. Cf. les travaux de Charles Tijus, laboratoire Cognitions humaine et artificielle de l'université Paris 8 (http://www.cognition-usages.org/chart2/).

\section{RÉSUMÉS}

Envisager la question du manifeste sous l'angle musical pose plusieurs problèmes, tant sur la définition de ce qui pourrait être qualifié de "musique révolutionnaire» que sur les déplacements que la musique pourrait en propre opérer dans ce que nous entendons par « révolution ». Après un retour critique sur le paradigme romantique qui renvoie la musique à un absolu poétique pour pouvoir lui reconnaître une force manifestaire, nous soulignerons le paradoxe sous-jacent aux révolutions dont les critères seraient si spécifiquement musicaux que leur portée resterait interne au domaine musical. Pour finir, nous renverrons l'hétéronomie 
musicale face à la revendication manifestaire aux conditions médiatiques de diffusion de la musique, jusqu'à considérer les tentatives de traduction des formes sonores par la «sémiotique temporelle » comme un éternel retour de l'esthétisation de la question révolutionnaire.

To consider the notion of manifesto from a musical angle raises several problems, both for the definition of what could qualify as "revolutionary music" and for the shift that music itself could perform in terms of our sense of the word "revolution." To begin with, we will critically examine the romantic paradigm which considers music as a poetic absolute in order to recognize its strength as a manifesto. Following which we will highlight the underlying paradox in revolutions whose criteria would be so specifically musical that their reach would remain internal to the musical domain. This will lead us to establish a link between the heteronomy of music towards the manifesto claim and the media conditions of music distribution, and consider the attempts to translate sound forms by "temporal semiotics" an eternal return of the aestheticisation of the revolutionary question.

INDEX

Keywords : music, revolution, manifesto, romanticism, aesthetics, philosophy

Mots-clés : musique, révolution, manifeste, romantisme, esthétique, philosophie

\section{AUTEUR}

DAVID CHRISTOFFEL

EHESS, CRAL (UMR 8566) 